OPEN ACCESS

Edited by:

Dongsheng Zhou,

Beijing Institute of Microbiology and Epidemiology, China

Reviewed by:

Charles William Stratton, Vanderbilt University Medical Center, United States Bum-Joon Kim Seoul National University, South Korea

*Correspondence: Laura Victoria laura.victoria0216@gmail.com

Specialty section: This article was submitted to

Clinical Microbiology,

a section of the journal

Frontiers in Cellular

and Infection Microbiology

Received: 28 January 2021 Accepted: 07 April 2021

Published: 26 April 2021

Citation:

Victoria L, Gupta A, Gómez JL and Robledo J (2021) Mycobacterium

abscessus complex: A Review of Recent Developments in an Emerging Pathogen.

Front. Cell. Infect. Microbiol. 11:659997. doi: 10.3389/fcimb.2021.659997

\section{Mycobacterium abscessus complex: A Review of Recent Developments in an Emerging Pathogen}

\author{
Laura Victoria $^{1,2 *}$, Amolika Gupta $^{3}$, Jose Luis Gómez ${ }^{3}$ and Jaime Robledo ${ }^{1,2}$ \\ 1 Laboratory of Bacteriology and Mycobacteria, Corporación para Investigaciones Biológicas (ClB), Medellín, Colombia, \\ ${ }^{2}$ Escuela de Ciencias de la Salud, Universidad Pontificia Bolivariana, Medellin, Colombia, ${ }^{3}$ Pulmonary, Critical Care and \\ Sleep Medicine Section, Yale University School of Medicine, New Haven, CT, United States
}

Mycobacterium abscessus complex (MABC) is one of the most clinically relevant species among nontuberculous mycobacteria. MABC's prevalence has increased over the last two decades. Although these changes can be explained by improvements in microbiological and molecular techniques for identifying species and subspecies, a higher prevalence of chronic lung diseases may contribute to higher rates of MABC. High rates of antimicrobial resistance are seen in MABC, and patients experience multiple relapses with low cure rates. This review aims to integrate existing knowledge about $\mathrm{MABC}$ epidemiology, microbiological identification and familiarize readers with molecular mechanisms of resistance and therapeutic options for pulmonary infections with MABC.

Keywords: Mycobacterium abscessus, lung, infection, resistance, treatment, epidemiology

\section{INTRODUCTION}

The prevalence of pulmonary infections caused by non-tuberculous mycobacteria (NTM) has increased over the past three decades (Kendall and Winthrop, 2013; Stout et al., 2016). In 1987, the US centers for disease control and prevention (CDC) estimated the NTM disease rate of 1.8/100.000 (Kendall and Winthrop, 2013). Data from North American studies between 2006 and 2012 suggested a disease rate of 5 to 10 per 100,000 (Prevots and Marras, 2015). Of the NTM causing pulmonary infections, Mycobacterium abscessus complex (MABC) is one of the most significant mycobacterial isolates associated with pulmonary infections (Schiff et al., 2019), particularly in patients with cystic fibrosis (Abdalla et al., 2015). Among the rapidly growing mycobacteria $(R G M)$, $M A B C$ is considered the most pathogenic of this group of pathogens (Medjahed et al., 2019). In particular, $M A B C$ is associated with intrinsic and acquired resistance to most anti-mycobacterial agents, including macrolides (Lopeman et al., 2019).

\section{Taxonomy}

Since $M A B C$ was first isolated in 1952 and was considered a new species of NTM (Lopeman et al., 2019), nomenclature has changed multiple times. In 1972, MABC was designated as M. chelonae subspecies abscessus but in 1992 thanks to DNA hybridization $M A B C$ was recognized as an independent species (Lee et al., 2015; Lopeman et al., 2019). In 2006, two new species $M$. massiliense and M. bolletii were described as novel and closely related to M. abscessus based on 
the rpoB gene sequence (Tortoli et al., 2016). However, since the three of them have more than $70 \%$ relatedness (based on DNADNA hybridization), M. massiliense, $M$. bolletii, and M. abscessus were presented as subspecies and the combinations of the three subspecies were known as Mycobacterium abscessus complex (MABC) (Tortoli et al., 2016).

Taxonomy classification has been controversial due to the significant similarities between $M$. massiliense and M. bolletii. In 2011, some authors considered that $M$. massiliense and $M$. bolettii should be classified as a single subspecies: $M$. abscessus subsp. bolletii. In 2013, whole-genome sequencingbased phylogenetic studies supported their differentiation in three distinct subspecies belonging to $M A B C$ (Lopeman et al., 2019). Additionally, the three subspecies are phenotypically divergent with clinically relevant impact which supports the importance of their differentiation as described by Tortoli et al., in 2016 (Tortoli et al., 2016; Lopeman et al., 2019). Currently, the three subspecies fulfill the criteria to be considered subspecies (Tortoli et al., 2018).

Recently, it was proposed to divide the genus Mycobacterium into 5 distinct genera: Mycobacterium, Mycolicibacterium, Mycolicibacter, Mycolicibacillus and Mycobacteroides. The basis for this division is the presence of specific molecular markers shared within a group of related taxa (Turenne, 2019). Mycobacteroides, proposed as new genus distinguished by 51 unique molecular markers, is also known as rapidly growing mycobacteroides and includes $M$. abscessus- $M$. chelonae clade (Gupta et al., 2018). The changed name of the genus proposed that affects the species name as Mycobacteroides abscessus, it is now considered a homotypic synonym of the species. Nevertheless, this approach is not widely yet and mycobacterium may continue to be used for $M A B$ taxonomy.

\section{Trends in Pulmonary Disease}

$M A B C$ is the most common etiological agent of pulmonary disease caused by RGM, representing 3-13\% of all NTM-PD (Honda et al., 2018). M. abscessus is the most common isolate, followed by M. massiliense and M. bolletii (Koh et al., 2014). However, the proportion of the three subspecies may vary according to geographical distribution (Koh et al., 2011).

In high burden areas of TB, NTM-PD has often been mistaken as multidrug-resistant tuberculosis (MDR-TB) (Shahraki et al., 2015). Patients treated for MDR-TB may have NTM disease (12-30\% of cases) (Nishiuchi et al., 2017). In a referral center in Brazil, $79 \%$ of patients had been treated empirically for TB for up to 6 months before the diagnosis of NTM disease was confirmed, evidencing a frequent misdiagnosis of NTM-PD (Prevots and Marras, 2015).

A systematic review that included 38,686 NTM isolates showed that $M A B C$ was frequently isolated in Asia (16\%) and Oceania (12\%) and less frequently in South America (5.7\%) North America (3.2\%) and Europe (2.9\%). Epidemiology updates of NTM reveal that the isolation frequency and clinical relevance of NTM in different world regions were MAC (88\%) M. Kansassii (78\%) and MABC (61\%) (Zweijpfenning et al., 2018).

\section{Environmental Sources}

NTM have been isolated from water tanks, hot tubes, swimming pools, medical devices such as endoscopes, seawater, house dust, and livestock, making exposure extremely common (Somoskovi, 2014; Halstrom et al., 2015; Loret and Dumoutier, 2019).

Environmental updates reveal that $M A B C$ is resistant to high chlorine levels that allows this mycobacterium to survive in drinking water (Jones et al., 2019). It is also resistant to disinfectants such as organic-mercurial substances and alkaline glutaraldehyde (Brown-Elliott and Wallace, 2002). MABC can form biofilms and resist high temperatures, which allow it to survive in water systems (Howard, 2013; Honda et al., 2018; Jones et al., 2019).

\section{Risk Factors For MABC-PD}

In patients with cystic fibrosis (CF), the estimated prevalence of $M A B C-P D$ is $13 \%$ in Europe and $16 \%$ in US (Harris and Kenna, 2014). Gastroesophageal disease, achalasia, and recurrent vomiting are conditions associated with recurrent aspiration of gastric contents, all reversible risk factors for $M A B C-P D$ (Jarand et al., 2011). Prior mycobacterial infection is a well-known risk factor for $M A B C-P D$ with previous or coexisting $M A C$ as high as 55.1\% (Jarand et al., 2011; Tung et al., 2015). Although there are no specific immune defects associated with $M A B C-P D$, autosomal recessive INF- $\gamma$ R1 deficiency has been described as a risk factor (Bustamante et al., 2014). A summary of related conditions is described in Table $\mathbf{1}$.

\section{Transmission}

There is no substantial evidence for person-to-person transmission or direct transmission from animals, but cases of infection occurring at the same location suggest a common source (Howard, 2013; Somoskovi, 2014; Bryant et al., 9877). Whole-genome sequencing (WGS) studies of outbreaks occurring in CF patients demonstrated clonality among isolates, suggesting that person-to-person transmission is a possible way of transmission (Howard, 2013). Additional evidence showed a few dominant clones spreading globally in the CF community (Ravnholt et al., 2018; Bryant et al., 9877). Transmission updates suggest that MABC is transmissible between CF patients in the hospital setting, including the transmission of macrolide-resistant isolates (Köser et al., 2014; Bryant et al., 9877). The finding that resistant strains might be transmissible has created a shift in our knowledge of MABC transmission (Ravnholt et al., 2018; Yoshida et al., 2018), and raised concerns about the clinical impact associated with global propagation of these strains.

\section{Immune Response, Virulence and Genomic Features}

Mycobacterial virulence depends on the ability of bacteria to invade, grow, and persist in the host. Type I interferons (IFN-I) such us IFN $\beta$ and IFN $\alpha$ play a critical role during $M A B C$ infection (Ruangkiattikul et al., 2019). Activation of toll-like receptors (TLR), specifically TLR2 and TLR4 results in MyD88/TRIF/IRF3 dependent IFN-I induction (Ruangkiattikul 
TABLE 1 | Risk factors associated with non-tuberculous mycobacterial pulmonary disease.

\begin{tabular}{|c|c|}
\hline NTM-PD ${ }^{a}$ & MABC-PD \\
\hline $\begin{array}{l}\text { Chronic obstructive pulmonary disease } \\
\text { Alpha-1-antitrypsin deficiency } \\
\text { Pneumoconiosis } \\
\text { Ciliary dyskinesia } \\
\text { Prior granulomatous disease } \\
\text { Thoracic skeletal abnormalities } \\
\text { Macrophage dysfunction } \\
\text { Biologic agents } \\
\text { Steroid use } \\
\text { CD4 lymphopenias } \\
\text { Hematologic malignancy } \\
\text { Mendelian susceptibility to NTM }{ }^{c}\end{array}$ & $\begin{array}{l}\text { Pre-existing pulmonary disease } \\
\text { Cystic fibrosis } \\
\text { Bronchiectasis } \\
\text { Gastroesophageal reflux } \\
\text { Achalasia } \\
\text { Lipoid pneumonia } \\
\text { Solid-organ cancer } \\
\text { History of Mycobacterial disease } \\
\text { (i.e. TB and NTM) }\end{array}$ \\
\hline $\begin{array}{l}\text { 'Multiple host susceptibility factors have be } \\
\text { Kasperbauer, 2019; van Ingen et al., 2019). } \\
\text { 'b Some risk factors appear to be highly asso } \\
{ }^{c} \text { Inherited conditions characterized by a preo } \\
\text { disseminated infections by species such as } \\
\text { multibacillary granulomas by M. abscessus, }\end{array}$ & $\begin{array}{l}\text { Stout et al., 2016; Drummond and } \\
\text { ficiency is associated with early-onset } \\
\text { ents with IFN- } \text { R2 deficiency present }\end{array}$ \\
\hline
\end{tabular}

et al., 2019; Peignier and Parker, 2021). IFN-I production in infected macrophages activates inducible nitric oxide synthase (NOS2) and nitric oxide (NO) production which can kill or induce dormancy. IFN-I plays a key role to induce NO production and intensify the ability of macrophages to clear MABC infection (Ruangkiattikul et al., 2019). Data suggest that persistence of $M A B C$ infections in CF patients could be explained by a limited IFN-I response. Interferon gamma (IFN- $\gamma$ ), a type II IFN is also required to control $M A B C$ infection, there have been several reports of disseminated disease in patients with defects in the IFN- $\gamma$ pathway (Rottman et al., 2007).

$M A B C$ induces a strong TLR2 mediated TNF $\alpha$ response, a key inflammatory cytokine that mediates mycobacterial killing, host defense and granuloma formation (Bernut et al., 2016; Kim et al., 2017). TNF/IL8 signaling pathway activates macrophage bactericidal activity, restrict extracellular growth, and increase neutrophil recruitment and mobilization which is required for granuloma formation (Bernut et al., 2016). It has also been shown that highly virulent isolates stimulate TNF secretion by macrophages (Medjahed et al., 2019). Impairment of this pathway correlates with disseminated disease and lethal infection (Bernut et al., 2016; Bernut et al., 2019). However, excessive levels of TNF-a can lead to detrimental effects in the host secondary to tissue damage (Kim et al., 2017).

$M A B C$ from clinical isolates have shown a rough vs smooth colony morphology and is able to shift between these forms (Medjahed et al., 2019). A zebrafish experimental model has been recently used for the study of $M A B C$ virulence, using this model, researchers observed that the rough morphology forms serpentine cords and large bacterial clumps, the formation of large cords allows this mycobacterium to escape the immune system as extremely large size of cords might prevent $M A B C$ from being internalized as they are larger in size than macrophages (Bernut et al., 2014; Bernut et al., 2017). This promotes spreads to other tissues and extracellular replication that results in abscess formation and tissue damage (Bernut et al.,
2014). The rough morphology is also associated with increased apoptosis leading to increases in extracellular bacteria and promotion of cord formation (Bernut et al., 2014). Interestingly, CFTR (CF transmembrane conductance regulator) defects, such as those seen in $\mathrm{CF}$, has been associated with impaired NADPH oxidase production which leads to increase intracellular growth and reduce neutrophil chemotaxis, compromising granuloma integrity (Bernut et al., 2019).

The absence of glycopeptidolipid (GLP), a molecule in the outer surface of the cell wall, has been associated with rough colony morphology. Defects in the mmpL4b gene are associated with the loss of GLP, leading to conversion to a rough phenotype showing morphological plasticity (Nessar et al., 2011; Bernut et al., 2016). The GLP loss unmask lipoproteins that produce a strong inflammatory response (Nessar et al., 2011). This correlates with the fact that hypervirulence has been observed in the rough morphology vs smooth morphology (Bernut et al., 2014). Rough morphology induces higher levels of TNF-I (Ruangkiattikul et al., 2019). Smooth morphology induces lower levels of IFN-I which favors persistence (Ruangkiattikul et al., 2019). Intramacrophage survival of the smooth morphology is also explained by phago-lysosomal fusion block and resistance to apoptosis, the phagosome shows membrane disruption at early stages of infection leading to phagosomecytosol communication and phagosomal escape allowing extracellular replication (Bernut et al., 2017). Phagosomal escape is independent of ESX-1 mechanism as MABC only has two ESX gene clusters (ESX-3 and ESX-4) which differs from TBC (Kim et al., 2017). Cord formation is a unique and new immune evasion mechanism in $M A B C$ infection.

The three subspecies of $M A B C$ are separated based on multilocus sequencing of housekeeping genes (Harris and Kenna, 2014). Unlike other $R G M, M A B C$ has a single ribosomal RNA operon, making the phenotypic expression of single mutation more likely. The reference strain (ATCC19977) contains a full genome sequence of 5.1 Megabases $(\mathrm{Mb})$ with a 
high number of conserved genes (Cortes et al., 2010; Medjahed et al., 2019). It includes an 81 - Kb full-length prophage, five insertion elements, and a $23 \mathrm{~Kb}$ - mercury resistance plasmid, which has been associated with infection in young patients with CF (Cortes et al., 2010). The resistant plasmid is highly similar to an episome present in M. Marinum, suggesting that these species may have exchanged the plasmid (Medjahed et al., 2019). MABC contains unique genes not present in other mycobacteria that appear to have been acquired by horizontal gene transfer from different species such as pseudomonas sp. and streptomyces $\mathrm{sp}$ (Howard, 2013; Medjahed et al., 2019).. These shared genes are thought to contribute to the pathogenesis of Pseudomonas sp. and $M A B C-P D$ facilitating respiratory tract colonization in $\mathrm{CF}$ patients (Nessar et al., 2011).

\section{Clinical Disease}

Updates reveal that there are two presentations of NTM pulmonary infections. One is an upper lobe fibrocavitary form while the other is a nodular bronchiectatic form (NB). The upper lobe fibrocavitary form is characterized by cavitary lesions similar to tuberculosis and usually occurs in older males with underlying lung disease (Shin et al., 2013; Moon et al., 2019). It is rapidly progressive and can lead to lung destruction in a short period of time (Sohn et al., 2009). The nodular bronchiectatic form is seen in the middle lobe of the right lung and lingula in the left lung. This form typically is present in nonsmoking postmenopausal women as bilateral bronchiectasis with small nodular opacities and tends to have a much slower course over time (Sohn et al., 2009; Moon et al., 2019). The natural course is usually indolent but progressive with a decline in pulmonary function, impaired quality of life and death in $15 \%$ of patients (Piersimoni and Scarparo, 2019). MABC infects patients with CF at an early age, and accounts for half of NTM isolates (Harris et al., 2012; Ravnholt et al., 2018). In CF patients, infection leads to an unexpected decline in lung function. This infection is particularly important in adolescents as it can cause the largest loss of potential years of life (Ravnholt et al., 2018).

\section{Resistance to Antibiotics}

Resistance updates reveal that the molecular mechanism responsible for macrolide resistance is the expression of an erythromycin ribosome methylase, erm gen. Other resistance molecular mechanisms include the rrs gene responsible for resistance in aminoglycosides, a class A beta-lactamase associated with resistance to most Beta-lactams (only cefoxitin and imipenem are useful), and enzymatic inactivation of most tetracyclines (except tigecycline). The principal mechanisms of antibiotic resistance are described in Table 2 .

The mechanism responsible for inducible macrolide resistance is the expression of an erythromycin ribosome methylase, erm(41) gene, in the presence of macrolides (Koh et al., 2014). This methylase transfers one or two methyl groups to adenine in the peptidyl region of 23S rRNA, preventing clarithromycin binding (Nessar et al., 2012; Pavan et al., 2017). M. massiliense harbors a truncated erm(41) gene being intrinsically susceptible to clarithromycin whereas $M$. abscessus contains a complete erm(41) conferring resistance, $M$. bolletti has the T28 polymorphism and may develop resistance to macrolides during therapy (Nessar et al., 2012; Kim et al., 2016; Pavan et al., 2017). Mutations in the $r r l$ gene encoding the 23 S rRNA peptidyl transferase are also associated with acquired macrolide resistance (Choi et al., 2017).

Spontaneous single mutations in the rrs gene encoding the 16S rRNA are responsible for resistance to aminoglycosides (Nessar et al., 2012). Nucleotide variation at the quinolone resistance determining region (QRDR) confers resistance to fluoroquinolones (Kim et al., 2016; Johansen et al., 2020). The genome of $M A B C$ encodes a class $\mathrm{A} \beta$-lactamase $\left(\mathrm{Bla}_{\mathrm{Mab}}\right)$ which is

TABLE 2 | Mechanism responsible for MABC antimicrobial resistance.

\begin{tabular}{|c|c|c|c|}
\hline ANTIMICROBIAL & $\begin{array}{l}\text { MECHANISMS OF } \\
\text { RESISTANCE }\end{array}$ & ENZYME/GENE & LOCATION \\
\hline Aminoglycosides & Target modifying enzymes & $\begin{array}{l}\text { Aminoglycoside2- } \mathrm{N} \text {-acetyltransferase and aminoglycosies } \\
\text { phosphotransferases }^{\mathrm{a}}\end{array}$ & \\
\hline $\begin{array}{l}\text { Deoxystreptamine } \\
\text { Aminoglycosides }\end{array}$ & $\begin{array}{l}\text { Acquired resistance by } \\
\text { point gene mutations }\end{array}$ & rrs gene encoding $16 \mathrm{~S}$ rRNA protein & $\begin{array}{l}\text { Mutations include T1406A, Cl409T, A1408G, and } \\
\text { G1491T. }^{\text {. }}\end{array}$ \\
\hline Beta Lactams & $\begin{array}{l}\text { Antibiotic degrading } \\
\text { enzymes }\end{array}$ & $\beta$-lactamase encoding genes. & Class A $\beta$-lactamase Bla_Mab (MAB_2875) \\
\hline Macrolides & $\begin{array}{l}\text { Target modifying enzymes } \\
\text { Acquired resistance by } \\
\text { point gene mutations }\end{array}$ & $\begin{array}{l}\text { Functioning erythromycin ribosome methylase erm(41) } \\
\text { gene } \\
\text { rrl gene encoding } 23 \mathrm{~S} \text { rRNA transferase }\end{array}$ & $\begin{array}{l}\text { Reversion to susceptibility: } 274 \text { bp deletion at } \\
\text { positions } 159-432 \text { and T28C point mutation. }{ }^{C} \\
\text { Point mutations at positions } 2058 \text { and } 2059\end{array}$ \\
\hline Fluoroquinolones & $\begin{array}{l}\text { Polymorphism in target } \\
\text { genes }\end{array}$ & $\begin{array}{l}\text { Nucleotide variation at the Quinolone resistance } \\
\text { determining region in the DNA gyrase- GyrA - GyrB }\end{array}$ & $\begin{array}{l}\text { Ala-90 gyrA gene } \\
\text { Arg-516 and Asp-533 gyrB gene }\end{array}$ \\
\hline Tetracyclines & Enzymatic inactivation & $\begin{array}{l}\text { Flavin- adenine- dinucleotide (FAD)- inactivating } \\
\text { monooxygenase (MabTetX) }\end{array}$ & \\
\hline \multicolumn{4}{|c|}{$\begin{array}{l}{ }^{a} \text { Twelve putative aminoglycoside phosphotransferases are encoded within the MABC genome, which could contribute to resistance to this group of antibiotics (Nessar et al., 2012; Luthra } \\
\text { et al., 2018). } \\
{ }^{b} \text { Mutations associated with aminoglycoside resistance (Ananta et al., 2018). } \\
{ }^{c} \text { These mechanisms are associated with reversion to clarithromycin susceptibility (Nie et al., 2014; Zhu et al., 2015). A T28C point mutation (thymidine to cytosine polymorphism at the } \\
\text { position 28) results in tryptophan to arginine amino acid change at codon 10, rendering a non-functional erm } 41 \text { gene (Pavan et al., 2017), C28 polymorphism is related to susceptibility } \\
\text { (Luthra et al., 2018). } \\
{ }^{d} \text { Quinolone resistance is associated with gyrA and gyrB mutations, a previous study showed all resistant isolates encoded the same amino acids in the quinolone resistance determining } \\
\text { region (Kim et al., 2016). }\end{array}$} \\
\hline
\end{tabular}


associated with resistance to most $\beta$-lactams. $M A B C$ hydrolyzes several members of cephalosporins and carbapenems, reducing its activity (Luthra et al., 2018; Story-roller et al., 2019). Cefoxitin and imipenem have moderate activity in vitro against $M A B C$ as they are hydrolyzed at a slow rate via Bla $_{\mathrm{Mab}}$ (Johansen et al., 2020).

Enzymatic inactivation of tetracyclines by the Flavinadenine- dinucleotide (FAD)- inactivating monooxygenase (MabTetX) has been reported (Rudra et al., 2018). This change is associated with high levels of resistance to doxycycline; however, tigecycline resists inactivation explaining its good activity in vitro (Luthra et al., 2018).

\section{Diagnosis}

Due to difficulties in differentiating between colonization from $M A B C$ isolates and true disease, clinical, radiologic, and microbiologic criteria are required for diagnosis (Ryu and Daley, 2016). The American Thoracic Society (ATS) and Infectious Diseases Society of America (IDSA) clinical guidelines for NTM diagnosis include these considerations.

A direct smear of the respiratory specimen should be examined using a fluorescent method with higher sensitivity than conventional stains like Ziehl-Neelsen; however, microscopy alone cannot distinguish between mycobacterial species and viable or non-viable specimens (Somoskovi, 2014). Several types of microbiologic culture systems have been used for diagnosis of RGM and $M A B C$, including growth indicator tube MGIT with BACTEC MGIT 960. Diagnostic updates suggest that culture is mandatory in all cases for diagnosis (Cortes et al., 2010). Growth is necessary for precise identification, with molecular methods being the gold standard for identification (Jones et al., 2019).

Despite identical 16S RNA genes, accurate identification can be achieved with the use of different targets that include RNA Polymerase ( $r p o B), g y r B$, heat shock protein ( $h s p 65)$, internal transcribed spacer (ITS), superoxide dismutase (sodA) and 16S 23S rRNA gene spacer amplification (Somoskovi, 2014; Jones et al., 2019). Mass spectrometry (MALDI- TOF) is capable of distinguishing between $M$. chelonae and $M A B C$ but cannot differentiate between the three subspecies of $M A B C$ (Jones et al., 2019).

Genotyping methods can be used to assess strain relatedness allowing outbreaks recognition. Commonly used methods are pulsed-field gel electrophoresis (PFGE), multilocus sequencing, and methods based on minisatellite sequences (variable number tandem repeats VNTR) and amplified fragment length polymorphism (Somoskovi, 2014). PFGE has been the standard method for differentiating strains within the complex (Howard, 2013). WGS offers a higher degree of resolution than other genotyping methods providing crucial information about transmission events (Harris and Kenna, 2014).

\section{Treatment}

Therapeutic updates suggest that in vitro antimicrobial testing in broth microdilution assay is required; incubation for 14 days is necessary to rule out inducible resistance for macrolides (Somoskovi, 2014; Jones et al., 2019).
Clinical and Laboratory Standards Institute (CLSI) guidelines recommend minimal inhibitory concentration (MICs) for susceptibility testing using a panel of 10 antimicrobials: amikacin, cefoxitin, clarithromycin, ciprofloxacin, doxycycline, imipenem, linezolid, moxifloxacin, trimethoprimsulfamethoxazole, and tobramycin (Koh et al., 2014).

$M A B C$ is one of the most resistant NTM, being uniformly resistant to conventional antituberculous drugs (Nessar et al., 2012; Howard, 2013). Cure rate is low, with success rates between 30 to $50 \%$ (Nie et al., 2014), except for M. massiliense (80 to $90 \%$ success rate) (Choi et al., 2017). A study on the clinical impact of differentiating $M$. massiliense and $M$. abscessus showed that clinical and radiological findings caused by the subspecies are very similar; however, the sputum conversion and maintenance of negative sputum was higher in patients with $M$. massiliense (88\%) pulmonary disease than M. abscessus (25\%) disease with overall negative cultures in 58\% of patients (Koh et al., 2011). Different sputum conversion rates could be explained by local differences in the prevalence of subspecies (Pasipanodya et al., 2017).

Treatment of $M A B C$ requires 18 months of multidrug therapy (Story-roller et al., 2019). Multidrug regimen includes macrolide based combination therapy with two parenteral agents for the initial phase (Jeong et al., 2017; Pasipanodya et al., 2017). Initial therapy should be given for at least 2-4 months, followed by oral macrolide based therapy (Koh et al., 2014; Lee et al., 2015). Recently, the British thoracic society has recommended for the initial phase to include 4 weeks of intravenous amikacin, tigecycline, imipenem (when tolerated) and oral clarithromycin. For the continuation phase, nebulized amikacin and an oral macrolide with one to three of the following: linezolid, clofazimine, minocycline cotrimoxazole, and moxifloxacin (Haworth et al., 2017). The goal is twelve-month sputum culture negativity (Thomson and Yew, 2009); However, recurrence and drug-related toxicity secondary to long term therapy are frequent, making this outcome unrealistic for many patients (Lyu et al., 2011; Koh et al., 2014). One study evaluating outcomes in patients with long term injectable therapy showed adverse effects in $43.9 \%$ of the patients, the most common was drug-induced liver injury (Lyu et al., 2011). Alternative goals include symptomatic improvement and radiographic regression of infiltrates rather than sputum conversion (Griffith et al., 2007; Koh et al., 2014).

A combination of oral clofazimine and inhaled amikacin demonstrated to be effective in refractory $M A B C-P D$ (Choi et al., 2017). Tigecycline combined with clarithromycin also appears synergistic against $M A B C(92.9 \%$ of patients with $M A B C$ infection). Additionally, tigecycline has shown good tissue penetration and few severe side effects making long-term therapy with this antibiotic an option (Huang et al., 2013). Other antibiotics such as linezolid showed good activity in clinical trials (Zhang et al., 2018). Rifabutin exhibits in vitro activity against $M A B C$ (Luthra et al., 2018). Bedaquiline is also active against $M A B C$ isolates (Johansen et al., 2020). A recent study showed synergy between non- $\beta$-lactam based inhibitors (avibactam, vaborbactam) with $\beta$-lactams, avibactam lowered the MICs for several $\beta$-lactams by 4-32 fold (Luthra et al., 2018). 
Recently, a study showed that clarithromycin-nanocapsules reduced colony numbers during $M A B C$ infection as they improved delivery of antimicrobial to mycobacteria inside macrophages (Anversa Dimer et al., 2020). NO has been also used as a promising strategy for treatment in patients with $M A B C$ infection and CF as NO kills bacterial cells and disperse biofilms avoiding resistance (Chiarelli et al., 2020). A young patient with $\mathrm{CF}$ and disseminated $M A B C$ infection was treated successfully with a three- phage cocktail therapy after lung transplantation (Dedrick et al., 2019). These new therapies represent promising strategies to overcome $M A B C$ resistance.

\section{CONCLUSION}

$M A B C$-PD has become a significant cause of pulmonary infection. When isolated, it is likely to represent a real infection. Precise identification to subspecies level is mandatory considering the differences in antibiotic susceptibility profiles and treatment outcomes; differentiation has become possible due to the development of new microbiological and molecular techniques. Macrolides are the

\section{REFERENCES}

Abdalla, M. Y., Ahmad, I. M., Switzer, B., and Britigan, B. E. (2015). Redox Biology Induction of Heme Oxygenase-1 Contributes to Survival of Mycobacterium Abscessus in Human Macrophages-Like THP-1 Cells. Redox Biol. 4, 328-339. doi: 10.1016/j.redox.2015.01.012

Ananta, P., Kham-ngam, I., Chetchotisakd, P., Chaimanee, P., Reechaipichitkul, W., Namwat, W., et al. (2018). Analysis of Drug-Susceptibility Patterns and Gene Sequences Associated With Clarithromycin and Amikacin Resistance in Serial Mycobacterium Abscessus Isolates From Clinical Specimens From Northeast Thailand. PloS One 13 (11), 1-15. doi: 10.1371/journal.pone. 0208053

Anversa Dimer, F., de Souza Carvalho-Wodarz, C., Goes, A., Cirnski, K., Herrmann, J., Schmitt, V., et al. (2020). PLGA Nanocapsules Improve the Delivery of Clarithromycin to Kill Intracellular Staphylococcus Aureus and Mycobacterium Abscessus. Nanomed. Nanotechnol. Biol. Med. 24, 102125. doi: 10.1016/j.nano.2019.102125

Bernut, A., Dupont, C., Ogryzko, N. V., Neyret, A., Herrmann, J. L., Floto, R. A., et al. (2019). Cftr Protects Against Mycobacterium Abscessus Infection by Fine-Tuning Host Oxidative Defenses. Cell Rep. 26 (7), 1828-1840.e4. doi: 10.1016/.j.celrep.2019.01.071

Bernut, A., Herrmann, J. L., Kissa, K., Dubremetz, J. F., Gaillard, J. L., Lutfalla, G., et al. (2014). Mycobacterium Abscessus Cording Prevents Phagocytosis and Promotes Abscess Formation. Proc. Natl. Acad. Sci. U. S. A. 111 (10), E943E952. doi: 10.1073/pnas.1321390111

Bernut, A., Herrmann, J. L., Ordway, D., and Kremer, L. (2017). The Diverse Cellular and Animal Models to Decipher the Physiopathological Traits of Mycobacterium Abscessus Infection. Front. Cell Infect. Microbiol. 7 (APR), 18. doi: 10.3389/fcimb.2017.00100

Bernut, A., Nguyen-Chi, M., Halloum, I., Herrmann, J. L., Lutfalla, G., and Kremer, L. (2016). Mycobacterium Abscessus-Induced Granuloma Formation Is Strictly Dependent on TNF Signaling and Neutrophil Trafficking. PloS Pathog. 12 (11), 1-28. doi: 10.1371/journal.ppat.1005986

Brown-Elliott, B. A., and Wallace, R. J. (2002). Clinical and Taxonomic Status of Pathogenic Nonpigmented or Late-Pigmenting Rapidly Growing Mycobacteria. Clin. Microbiol. Rev. 15 (4), 716-746. doi: 10.1128/ CMR.15.4.716-746.2002

Bryant, J. M., Grogono, D. M., Greaves, D., Foweraker, J., Roddick, I., Inns, T., et al. (9877). Whole-Genome Sequencing to Identify Transmission of Mycobacterium mainstay of therapy; therefore, identification of inducible resistance strains is necessary before starting treatment. $M A B C$ is considered a multidrug-resistant pathogen, and increasing resistance is highly likely to continue given the limited existing therapeutic options. Consequently, it is important to develop novel therapies and identify combinations of currently available antimicrobials to improve treatment outcomes.

\section{AUTHOR CONTRIBUTIONS}

LV, AG, JG, and JR conceived of the present idea and performed literature review. LV wrote the manuscript with the support from JG and JR. All authors contributed to the article and approved the submitted version.

\section{ACKNOWLEDGMENTS}

The authors would like to thank Coporacion para Investigaciones Biológicas and Yale University School of medicine for their support.

Abscessus Between Patients With Cystic Fi Brosis : A Retrospective Cohort Study. Lancet 381, 1551-1560. doi: 10.1016/S0140-6736(13)60632-7

Bustamante, J., Boisson-Dupuis, S., Abel, L., and Casanova, J. L. (2014). Mendelian Susceptibility to Mycobacterial Disease: Genetic, Immunological, and Clinical Features of Inborn Errors of IFN- $\gamma$ Immunity. Semin. Immunol. 26 (6), 454470. doi: 10.1016/j.smim.2014.09.008

Chiarelli, L. R., Degiacomi, G., Egorova, A., Makarov, V., and Pasca, M. R. (2020). Nitric Oxide-Releasing Compounds for the Treatment of Lung Infections. Drug Discovery Today 26 (2), 542-550.. doi: 10.1016/j.drudis.2020.10.027

Choi, H., Kim, S.-Y., Kim, D. H., Huh, H. J., Ki, C.-S., Lee, N. Y., et al. (2017). Clinical Characteristics and Treatment Outcomes of Patients With Acquired Macrolide-Resistant Mycobacterium Abscessus Lung Disease. Antimicrob. Agents Chemother. 61 (10), 1-10. doi: 10.1128/AAC.01146-17

Cortes, M. A. M., Nessar, R., and Singh, A. K. (2010). Laboratory Maintenance of Mycobacterium Abscessus. Curr. Protoc. Microbiol. Chapter 10, Unit 10D.1.. doi: 10.1002/9780471729259.mc10d01s18

Dedrick, R. M., Guerrero-Bustamante, C. A., Garlena, R. A., Russell, D. A., Ford, K., Harris, K., et al. (2019). Engineered Bacteriophages for Treatment of a Patient With a Disseminated Drug-Resistant Mycobacterium Abscessus. Nat. Med. 25 (5), 730-733. doi: 10.1038/s41591-019-0437-z

Drummond, W. K., and Kasperbauer, S. H. (2019). Nontuberculous Mycobacteria: Epidemiology and the Impact on Pulmonary and Cardiac Disease. Thorac. Surg. Clin. 29 (1), 59-64. doi: 10.1016/j.thorsurg.2018.09.006

Griffith, D. E., Aksamit, T., Brown-Elliott, B. A., Catanzaro, A., Daley, C., Gordin, F., et al. (2007). An Official ATS/IDSA Statement: Diagnosis, Treatment, and Prevention of Nontuberculous Mycobacterial Diseases. Am. J. Respir. Crit. Care Med. 175 (4), 367-416. doi: 10.1164/rccm.200604-571ST

Gupta, R. S., Lo, B., and Son, J. (2018). Phylogenomics and Comparative Genomic Studies Robustly Support Division of the Genus Mycobacterium Into an Emended Genus Mycobacterium and Four Novel Genera. Front. Microbiol. 9 (FEB), 1-41. doi: 10.3389/fmicb.2018.00067

Halstrom, S., Price, P., and Thomson, R. (2015). Review: Environmental Mycobacteria as a Cause of Human Infection. Int. J. Mycobacteriol. 4 (2), 81-91. doi: 10.1016/j.ijmyco.2015.03.002

Harris, K. A., and Kenna, D. T. D. (2014). Mycobacterium Abscessus Infection in Cystic Fibrosis: Molecular Typing and Clinical Outcomes. J. Med. Microbiol. 63 (2014), 1241-1246. doi: 10.1099/jmm.0.077164-0

Harris, K. A., Kenna, D. T. D., Blauwendraat, C., Hartley, J. C., Turton, J. F., Aurora, P., et al. (2012). Molecular Fingerprinting of Mycobacterium 
Abscessus Strains in a Cohort of Pediatric Cystic Fibrosis Patients. J. Clin. Microbiol. 50 (5), 1758-1761. doi: 10.1128/JCM.00155-12

Haworth, C. S., Banks, J., Capstick, T., Fisher, A. J., Gorsuch, T., Laurenson, I. F., et al. (2017). British Thoracic Society Guideline for the Management of nonTuberculous Mycobacterial Pulmonary Disease (NTM-PD). BMJ Open Respir. Res. 4 (1), e000242. doi: 10.1136/bmjresp-2017-000242

Honda, J. R., Virdi, R., and Chan, E. D. (2018). Global Environmental Nontuberculous Mycobacteria and Their Contemporaneous Man-Made and Natural Niches. Front. Microbiol. 9 (AUG), 1-11. doi: 10.3389/ fmicb.2018.02029

Howard, S. T. (2013). Recent Progress Towards Understanding Genetic Variation in the Mycobacterium Abscessus Complex. Tuberculosis 93, S15-S20. doi: 10.1016/S1472-9792(13)70005-2

Huang, C. W., Chen, J. H., Hu, S. T., Huang, W. C., Lee, Y. C., Huang, C. C., et al. (2013). Synergistic Activities of Tigecycline With Clarithromycin or Amikacin Against Rapidly Growing Mycobacteria in Taiwan. Int. J. Antimicrob. Agents 41 (3), 218-223. doi: 10.1016/j.ijantimicag.2012.10.021

Jarand, J., Levin, A., Zhang, L., Huitt, G., Mitchell, J. D., and Daley, C. L. (2011). Clinical and Microbiologic Outcomes in Patients Receiving Treatment for Mycobacterium Abscessus Pulmonary Disease. Clin. Infect. Dis. 52 (5), 565571. doi: $10.1093 / \mathrm{cid} / \mathrm{ciq} 237$

Jeong, S. H., Kim, S. Y., Huh, H. J., Ki, C. S., Lee, N. Y., Kang, C. I., et al. (2017). Mycobacteriological Characteristics and Treatment Outcomes in Extrapulmonary Mycobacterium Abscessus Complex Infections. Int. J. Infect. Dis. 60, 49-56. doi: 10.1016/j.ijid.2017.05.007

Johansen, M. D., Herrmann, J. L., and Kremer, L. (2020). Non-Tuberculous Mycobacteria and the Rise of Mycobacterium Abscessus. Nat. Rev. Microbiol. 18, 392-407. doi: 10.1038/s41579-020-0331-1

Jones, R. S., Shier, K. L., Master, R. N., Bao, J. R., and Clark, R. B. (2019). Current Significance of the Mycobacterium Chelonae-Abscessus Group. Diagn. Microbiol. Infect. Dis. 94 (3), 248-254. doi: 10.1016/j.diagmicrobio.2019.01.021

Kendall, B. A., and Winthrop, K. L. (2013). Update on the Epidemiology of Pulmonary Nontuberculous Mycobacterial Infections. Semin. Respir. Crit. Care Med. 34 (1), 87-94. doi: 10.1055/s-0033-1333567

Kim, J., Sung, H., Park, J. S., Choi, S. H., Shim, T. S., and Kim, M. N. (2016). Subspecies Distribution and Macrolide and Fluoroquinolone Resistance Genetics of Mycobacterium Abscessus in Korea. Int. J. Tuberc. Lung Dis. 20 (1), 109-114. doi: 10.5588/ijtld.15.0068

Kim, Y. S., Yang, C. S., Nguyen, L. T., Kim, J. K., Jin, H. S., Choe, J., et al. (2017). Mycobacterium Abscessus ESX-3 Plays an Important Role in Host Inflammatory and Pathological Responses During Infection. Microbes Infect. 19 (1), 5-17. doi: 10.1016/j.micinf.2016.09.001

Koh, W. J., Jeon, K., Lee, N. Y., Kim, B. J., Kook, Y. H., Lee, S. H., et al. (2011). Clinical Significance of Differentiation of Mycobacterium Massiliense From Mycobacterium Abscessus. Am. J. Respir. Crit. Care Med. 183 (3), 405-410. doi: 10.1164/rccm.201003-0395OC

Koh, W. J., Stout, J. E., and Yew, W. W. (2014). Advances in the Management of Pulmonary Disease Due to Mycobacterium Abscessus Complex. Int. J. Tuberc. Lung Dis. 18 (10), 1141-1148. doi: 10.5588/ijtld.14.0134

Köser, C. U., Ellington, M. J., and Peacock, S. J. (2014). Whole-Genome Sequencing to Control Antimicrobial Resistance. Trends Genet. 30 (9), 401407. doi: 10.1016/j.tig.2014.07.003

Lee, M.-R., Sheng, W.-H., Hung, C.-C., Yu, C.-J., Lee, L.-N., and Hsueh, P.-R. (2015). Mycobacterium Abscessus Complex Infections in Humans. Emerg. Infect. Dis. 21 (9), 1638-1646. doi: 10.3201/2109.141634

Lopeman, R. C., Harrison, J., Desai, M., and Cox, J. A. G. (2019). Mycobacterium Abscessus: Environmental Bacterium Turned Clinical Nightmare. Microorganisms 7 (3), 90. doi: 10.3390/microorganisms7030090

Loret, J., and Dumoutier, N. (2019). International Journal of Hygiene and Nontuberculous Mycobacteria in Drinking Water Systems : A Review of Prevalence Data and Control Means. Int. J. Hyg. Environ. Health 222 (4), 628-634. doi: 10.1016/j.ijheh.2019.01.002

Luthra, S., Rominski, A., and Sander, P. (2018). The Role of Antibiotic-TargetModifying and Antibiotic-Modifying Enzymes in Mycobacterium Abscessusdrug Resistance. Front. Microbiol. 9 (SEP), 1-13. doi: 10.3389/ fmicb.2018.02179

Lyu, J., Jang, H. J., Song, J. W., Choi, C. M., Oh, Y. M., Do, L. S., et al. (2011). Outcomes in Patients With Mycobacterium Abscessus Pulmonary Disease
Treated With Long-Term Injectable Drugs. Respir. Med. 105 (5), 781-787. doi: 10.1016/j.rmed.2010.12.012

Medjahed, H., Gaillard, J., and Reyrat, J. (2019). Mycobacterium Abscessus : A New Player in the Mycobacterial Field. Trends Microbiol. 18 (3), 117-123. doi: 10.1016/j.tim.2009.12.007

Moon, S. M., Jhun, B. W., Baek, S. Y., Kim, S., Jeon, K., Ko, R. E., et al. (2019). Long-Term Natural History of non-Cavitary Nodular Bronchiectatic Nontuberculous Mycobacterial Pulmonary Disease. Respir. Med. 151, 1-7. doi: 10.1016/j.rmed.2019.03.014

Nessar, R., Cambau, E., Reyrat, J. M., Murray, A., and Gicquel, B. (2012). Mycobacterium Abscessus: A New Antibiotic Nightmare. J. Antimicrob. Chemother. 67 (4), 810-818. doi: 10.1093/jac/dkr578

Nessar, R., Reyrat, J. M., Davidson, L. B., and Byrd, T. F. (2011). Deletion of the mmpL4b Gene in the Mycobacterium Abscessus Glycopeptidolipid Biosynthetic Pathway Results in Loss of Surface Colonization Capability, But Enhanced Ability to Replicate in Human Macrophages and Stimulate Their Innate Immune Response. Microbiology 157 (4), 1187-1195. doi: 10.1099/ mic. $0.046557-0$

Nie, W., Duan, H., Huang, H., Lu, Y., Bi, D., and Chu, N. (2014). Species Identification of Mycobacterium Abscessus Subsp. Abscessus and Mycobacterium Abscessus Subsp. Bolletii Using Rpob and hsp65, and Susceptibility Testing to Eight Antibiotics. Int. J. Infect. Dis. 25, 170-174. doi: 10.1016/j.ijid.2014.02.014

Nishiuchi, Y., Iwamoto, T., and Maruyama, F. (2017). Infection Sources of a Common non-Tuberculous Mycobacterial Pathogen, Mycobacterium Avium Complex. Front. Med. 4, 27. doi: 10.3389/fmed.2017.00027

Pasipanodya, J. G., Ogbonna, D., Ferro, B. E., Magombedze, G., Srivastava, S., Deshpande, D., et al. (2017). Systematic Review and Meta-Analyses of the Effect of Chemotherapy on Pulmonary Mycobacterium Abscessus Outcomes and Disease Recurrence. Antimicrob. Agents Chemother. 61 (11), 1-14. doi: 10.1128/AAC.01206-17

Pavan, F., Chimara, E., Leite, C. Q. F., Arbeit, R. D., Sato, D. N., and de Carvalho, N. F. G. (2017). Genetic Correlates of Clarithromycin Susceptibility Among Isolates of the Mycobacterium Abscessus Group and the Potential Clinical Applicability of a PCR-based Analysis of Erm(41). J. Antimicrob. Chemother. 73 (4), 862-866. doi: 10.1093/jac/dkx476

Peignier, A., and Parker, D. (2021). Impact of Type I Interferons on Susceptibility to Bacterial Pathogens. Trends Microbiol. 1-13. doi: 10.1016/j.tim.2021.01.007

Piersimoni, C., and Scarparo, C. (2019). Pulmonary Infections Associated With Non-Tuberculous Mycobacteria in Immunocompetent Patients . Lancet Infect. Dis. 8 (5), 323-334. doi: 10.1016/S1473-3099(08)70100-2

Prevots, D. R., and Marras, T. K. (2015). Epidemiology of Human Pulmonary Infection With Nontuberculous Mycobacteria: A Review. Clin. Chest Med. 36 (1), 13-34. doi: 10.1016/j.ccm.2014.10.002

Ravnholt, C., Kolpen, M., Skov, M., Moser, C., Katzenstein, T. L., Pressler, T., et al. (2018). The Importance of Early Diagnosis of Mycobacterium Abscessus Complex in Patients With Cystic Fibrosis. Apmis 126 (12), 885-891. doi: 10.1111/apm.12903

Rottman, M., Catherinot, E., Hochedez, P., Emile, J. F., Casanova, J. L., Gaillard, J. L., et al. (2007). Importance of T Cells, Gamma Interferon, and Tumor Necrosis Factor in Immune Control of the Rapid Grower Mycobacterium Abscessus in C57BL/6 Mice. Infect. Immun. 75 (12), 5898-5907. doi: 10.1128/IAI.00014-07

Ruangkiattikul, N., Rys, D., Abdissa, K., Rohde, M., Semmler, T., Tegtmeyer, P. K., et al. (2019). Type I Interferon Induced by TLR2-TLR4-MyD88-TRIF-IRF3 Controls Mycobacterium Abscessus Subsp. Abscessus Persistence in Murine Macrophages Via Nitric Oxide. Int. J. Med. Microbiol. 309 (5), 307-318. doi: 10.1016/j.ijmm.2019.05.007

Rudra, P., Hurst-Hess, K., Lappierre, P., and Ghosha, P. (2018). High Levels of Intrinsic Tetracycline Resistance in Mycobacterium Abscessus are Conferred by a Tetracycline-Modifying Monooxygenase. Antimicrob. Agents Chemother. 62 (6), 1-14. doi: 10.1128/AAC.00119-18

Ryu, Y. J., and Daley, C. L. (2016). Diagnosis and Treatment of NTM Lung Diseases Diagnosis of NTM Lung Disease. Tuberc. Respir. Dis. 79, 74-84. doi: 10.4046/trd.2016.79.2.74

Schiff, H. F., Jones, S., Achaiah, A., Pereira, A., Stait, G., and Green, B. (2019). Clinical Relevance of non-Tuberculous Mycobacteria Isolated From Respiratory Specimens: Seven Year Experience in a UK Hospital. Sci. Rep. 9 (1), 6-11. doi: 10.1038/s41598-018-37350-8 
Shahraki, A. H., Heidarieh, P., Bostanabad, S. Z., Khosravi, A. D., Hashemzadeh, M., Khandan, S., et al. (2015). "Multidrug-Resistant Tuberculosis" may be Nontuberculous Mycobacteria. Eur. J. Intern. Med. 26 (4), 279-284. doi: 10.1016/j.ejim.2015.03.001

Shin, S. J., Choi, G.-E., Cho, S.-N., Woo, S. Y., Jeong, B.-H., Jeon, K., et al. (2013). Mycobacterial Genotypes are Associated With Clinical Manifestation and Progression of Lung Disease Caused by Mycobacterium Abscessus and Mycobacterium Massiliense. Clin. Infect. Dis. 57 (1), 32-39. doi: 10.1093/cid/ cit172

Sohn, H., Kim, H., Man, J., Kwon, O. J., Koh, W., and Jae, S. (2009). Microbial Pathogenesis High Virulent Clinical Isolates of Mycobacterium Abscessus From Patients With the Upper Lobe Fibrocavitary Form of Pulmonary Disease. Microb. Pathog. 47 (6), 321-328. doi: 10.1016/j.micpath.2009.09.010

Somoskovi, A. (2014). Nontuberculous Mycobacteria in Respiratory Infections. Clin. Lab. Med. 34 (2), 271-295. doi: 10.1016/j.cll.2014.03.001

Story-Roller, E., Maggioncalda, E. C., and Lamichhane, G. (2019). Select $\beta$-Lactam Combinations Exhibit Synergy against Mycobacterium abscessus In Vitro. Antimicrob Agents Chemother. 63 (4), e02613-18. doi: 10.1128/AAC.02613-18

Stout, J. E., Koh, W. J., and Yew, W. W. (2016). Update on Pulmonary Disease Due to non-Tuberculous Mycobacteria. Int. J. Infect. Dis. 45, 123-134. doi: 10.1016/ j.ijid.2016.03.006

Thomson, R. M., and Yew, W. W. (2009). When and How to Treat Pulmonary Non-Tuberculous Mycobacterial Diseases. Respirology 14 (1), 12-26. doi: 10.1111/j.1440-1843.2008.01408.x

Tortoli, E., Kohl, T. A., Brown-Elliott, B. A., Trovato, A., Cardoso-Leão, S., Garcia, M. J., et al. (2018). Mycobacterium Abscessus, a Taxonomic Puzzle. Int. J. Syst. Evol. Microbiol. 68 (1), 467-469. doi: 10.1099/ijsem.0.002457

Tortoli, E., Kohl, T. A., Brown-Elliott, B. A., Trovato, A., Leão, S. C., Garcia, M. J., et al. (2016). Emended Description of Mycobacterium Abscessus Mycobacterium Abscessus Subsp. Abscessus and Mycobacterium Abscessus Subsp. Bolletii and Designation of Mycobacterium Abscessus Subsp. Massiliense Comb. Int. J. Syst. Evol. Microbiol. 66 (11), 4471-4479. doi: 10.1099/ijsem.0.001376

Tung, Y. J., Bittaye, S. O., Tsai, J. R., Lin, C. Y., Huang, C. H., Chen, T. C., et al. (2015). Risk Factors for Microbiologic Failure Among Taiwanese Adults With
Mycobacterium Abscessus Complex Pulmonary Disease. J. Microbiol. Immunol. Infect. 48 (4), 437-445. doi: 10.1016/j.jmii.2014.08.005

Turenne, C. Y. (2019). Nontuberculous Mycobacteria: Insights on Taxonomy and Evolution. Infect. Genet. Evol. 72 (November 2018), 159-168. doi: 10.1016/ j.meegid.2019.01.017

van Ingen, J., De Zwaan, R., Dekhuijzen, R. P. N., Boeree, M. J., and van Soolingen, D. (2019). Clinical Relevance of Mycobacterium Chelonae E Abscessus Group Isolation in 95 Patients. J. Infect. 59 (5), 324-331. doi: 10.1016/ j.jinf.2009.08.016

Yoshida, M., Fukano, H., Miyamoto, Y., Shibayama, K., and Suzuki, M. (2018). Crossm Complete Genome Sequence of a Type Strain of Mycobacterium Abscessus Complex Genome Announc. 6 (5), e01530-17. doi: 10.1128/ genomeA.01530-17

Zhang, Z., Lu, J., Song, Y., and Pang, Y. (2018). In Vitro Activity Between Linezolid and Other Antimicrobial Agents Against Mycobacterium Abscessus Complex. Diagn. Microbiol. Infect. Dis. 90 (1), 31-34. doi: 10.1016/j.diagmicrobio.2017.09.013

Zhu, Y. C., Mitchell, K. K., Nazarian, E. J., Escuyer, V. E., and Musser, K. A. (2015). Rapid Prediction of Inducible Clarithromycin Resistance in Mycobacterium Abscessus. Mol. Cell Probes 29 (6), 514-516. doi: 10.1016/j.mcp.2015.08.007

Zweijpfenning, S. M. H., van Ingen, J., and Hoefsloot, W. (2018). Geographic Distribution of Nontuberculous Mycobacteria Isolated From Clinical Specimens: A Systematic Review. Semin. Respir. Crit. Care Med. 39 (3), 336 342. doi: 10.1055/s-0038-1660864

Conflict of Interest: The authors declare that the research was conducted in the absence of any commercial or financial relationships that could be construed as a potential conflict of interest.

Copyright (c) 2021 Victoria, Gupta, Gómez and Robledo. This is an open-access article distributed under the terms of the Creative Commons Attribution License (CC BY). The use, distribution or reproduction in other forums is permitted, provided the original author(s) and the copyright owner(s) are credited and that the original publication in this journal is cited, in accordance with accepted academic practice. No use, distribution or reproduction is permitted which does not comply with these terms. 